maximum geringer als das entsprechende Verhältnis für Brom.

6. Chtor verhält sich unter den gleichen Bedingungen gänzlich verschieden von den beiden anderen Halogenen. Adsorptionserscheinungen konnten nicht wahrgenommen werden, sondern die Resultate weisen auf die Entstehung einer chemischen Verbindung bin.

7. Geringe Differenzen in der Trockenheit des Kalkes haben auf die Adsorption von Brom und Jod einen größeren Einfluß als auf die von Chlor.

Zum Schlusse möchte ich Herrn Dr. Fent on bestens danken, sowohl fiir die Anregung zu dieser Arbeit, als auch für das stete Interesse und die Unterstïtzung, die er derselben zuteil werden liek

The University Chemical Laboratory Cambridge.

\title{
Notizen über die Adsorption.
}

Von L. Gurwitsch (St. Petersburg).

(Eingegangen am 2. Juni 1912)

Gelegentlich einer Untersuchung über die Einwirkung von Entfärbungspulvern auf Erdölprodukte habe ich einige Beobachtungen gemacht, die vielleicht von allgemeinem Interesse sein dürften und die ich deshalb an dieser Stelle mitteile.

\section{Einfluß der Korngröße.}

Um den Einflub verschiedener Korngröben des Adsorbens auf den Verlauf der Adsorption kennen $z u$ lernen, wurde einerseits das ziemlich grobkörnige „Floridin S“ (KorngröBe durchschnittlich etwa $0,8 \mathrm{~mm}$ ), andererseits dasselbe Floridin in feinst zerriebener Form (Korngröße etwa $0,002 \mathrm{~mm}$ ) genommen $\%$. Der Verlauf der Adsorption wurde an Lósungen voll sogenanntem Celgondron ${ }^{2}$ in Benzin st:idiert. Für jedo Bestimmung war eine neue Portion derselben Lösung genommen und wabrend einer bestimmten Zeit mit demselben Prozentsat\% Floridin auf der Schüttelmaschine behandelt worden. Die beinandelte Benzinlosung wurde möglichst schnell in einem Allihn'schen geschlossenen Apparat filtriert und der Gehalt an Oelgoudron durch Verdampfen eines aliquoten Teils in Kohiensäureatmosphäre bis zum konstanten Gewicht bestimmt. Bci Wiederholung mehrerer Versuche differierten die erhaltenen Zahlen nicht mehr als um $0,01-0,02$. Der zeitliche Verlauf der Adsorption war nun folgender (Tabelle I).

1) Um den EinfluB der möglicherweise verschiedenen Röstung resp. verschiedenen Feuchtigkeits. gehalts zu eliminieren, wurde Floridin "S* frisch gerostet, ein Tell davon zerrieben und bei $150^{\circ}$ getrocknet.

2) Erdölrückstand nach Abtreiben aller Fraktionen inklusive Zylinderöle; besteht aus sehr hoclimolekularen und scliwerflussigen Kohlenwasserstoffen, sowie Harz- und Asphaltstoffen usw.
Tabelle I

Adsorbierte Menge pro 100 Teile Floridin

\begin{tabular}{|c|c|c|}
\hline & feinkörnig & grobkörnig \\
\hline 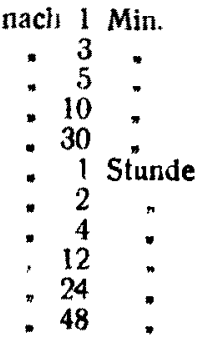 & $\begin{array}{l}1,74 \\
2,48 \\
3,00 \\
3,40 \\
4,22 \\
4,58 \\
4,72 \\
4,77 \\
4,80 \\
4,81 \\
4,83\end{array}$ & $\begin{array}{l}2,71 \\
3,10 \\
3,55 \\
4,02 \\
4,68 \\
4,75 \\
4,78 \\
4,80 \\
4,81 \\
4,82 \\
4,82\end{array}$ \\
\hline
\end{tabular}

Man sieht somit, dak die Adsorption mit feinem Floridin bedeutend schneller als mit dem groben abläuft, daß aber der Grenzzustand in beiden Fälen derselbe ist. In Anbetracht der so sehr verschiedenen Korngr $\measuredangle B$ Be muß daraus geschiossen werden, daß das Floridin nicht nur in gewöhnlichem Sinne porös, sondern in seiner ganzen Masse mit mikroskopischen und ultramikroskopischen Gängen durchsetzk ist. Ja, es erscheint sogar die Annahme plausibel, dat es eine mizellase oder dergleichen innere Struktur besitzt und daB die Adsorption der Quellung der Kolloide insofern an die Scite zu stellen is!, als man es auch hier mit einer ganz intimen Durchdringung des Adsorbens durch die adsorbierten Stoffe zu tun hat.

Da der Oelgoudron keine einheitliche Sub. stanz, sondern ein Gemisch vieler verschiedener Stoffe carstellt, sind, neben den Veränderungen des Gesamtgehaltes des nicht flüchtigen, auch die Färbungsgrade der mit Floridin behandelten Lösungen bestimmt worden (Tabelle II). 
Tabelle II

Farbe $^{3}$ ) der Lösung nach Behandlung miı Floridin

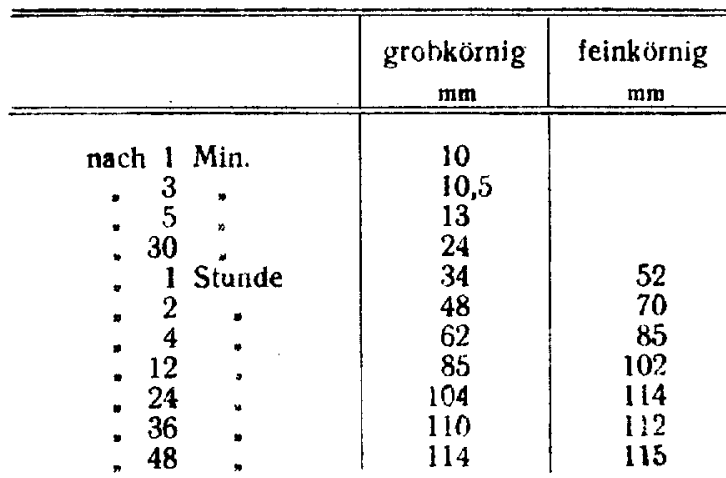

Man findet wiederum, daß die Entfürbung mit dem feinen Fioridin schneller, als nit dem grobkörnigen vor sich geht, daB aber der $z u$ erreichende Grenzzustand mit beiden derselbe ist. Der Vergleich der Zahlen für die Entfärbming und die Gesamladsorption führt aber zu einem weiteren interessanten Resultat. Man sieht nämlich, dab sowoh! mit grobem, wie mit feinem Ploridin die Eutfärbung langsamer. als die Gesamtadsorption verläuft. Da es nun höchstwahrscheinlich ist, dals die eigentliche Adsorption, sobald eben die zu adsorbierende Substanz mil dem Adsorbens in direkte Berührung gekommen ist, sehr schnell vor sich geht, kann das verschiedene Tempo der Gesamtadsorption und der Entfärbung ain ehesten auf folgende Weise gedeutet werden. Die in das Innere der Floridinsubstanz zuerst eindringende Lösung ist schon an den 711 adsorbierender Stoffen verarm, da diese an der äuBeren Oberfläche der Ploridinkörner zurückgehalten werden.

Es findet daher ein Diffusionsstrom der zu adsorbierenden Stoffe aus der übrigen 1 ösung in das Innere des Floridins statt; je langsamer diese Diffusion vor sich geht, um so länger dauert es, bis der Grenzzustand der Diffusion erreicht wird, und die Unterschiede im Diffusions vermögen verschiedener Stoffe bewirken eben auch Unterschiede in der Geschwindigkeit ihrer Adsorption. Würde dagegen das langsame Einstellen der Adsorption, wie es G. Schmidi annimmt ${ }^{4}$ ), dadurch bedingt sein, dab die Kanälchen und Hohlräume des Adsorbens mit Luft gefüllt sind, welche den Zutritt der Flüssigkeit in diese Hohlräume verhindert, so daß die Flüssigkeit in das Innere des Adsorbens nur allmählich.

8) Die Farbe ist mit einem $S$ tammer'schen Kolorimeter mit einenl willkürlich gewăhiten Farbglase bestimmt worden.

1. G. Schmidt, Zeitschr. f. physik. Chem. 74, 689 nach Mafigabe des Auflösens der Luft hineindringt, so müBten alle in derselben Lösung befindlichen Stoffe denselben zeitlichen Verlauf der Adsorption aufweisen.

\section{Adsorption von Paraffin.}

Die Auflösung von Paraffin in organischen Flüssigkeiten geschieht unter ziemlich bedeutender Ausdehnung des ganzen Systems; so z. B. fand E. Graefe ${ }^{5}$ ) beim Auflösen von $100 \mathrm{~g}$ Paraffin in 1 Liter Benzol eine Volumzunahme von $10,8 \mathrm{ccm}$. Nach dem Prinzip von $\mathrm{Braun}-\mathrm{L}$ e $\mathrm{Chatelier}$ muB somit die Löslichkeit von Paraffin in Benzol mit wachsendem Drucke abnehmen; dann aber sollte nach der Adsorptionshypothese von S. Lagergreen die Adsorption von Paraffin eine negative sein. Durch einen direkten Versuch habe ich nun das Gegenteil gefunden : aus einer 10 prozentigen Benzollösung wurden von 20 Proz. Floridin (auf die Lösung gerechnet) 1,64 Proz. Paraffin adsorbiert. Was also gegen die Lagergreen'sche Hypothese spricht.

\section{Polymerisierende Wirkungen.}

Es ist bisher, soviel ich weib, kein Fall einer Polymerisation unter der Einwirkung von porösen Körpern bei gewöhnlicher Temperatur beobachtet worden. Einen solchen habe ich nun bei der Behandlung von Amylen mit Hydrosilikaten gefunden. Es wurde mit frisch umrektifiziertem, zwischen $39-40,5^{\circ}$ siedendem Amylen (spezifisches Gewicht $0,6654^{170}$ ) gearbeitet. Merkwürdig grob war die Wärmeent wicklung beim Zusammenbringen des Amylens mit Floridin; beim Vermischen gleicher Teile beider in eincin Dewar'schen Gefäß stieg die Temperalur von $17^{\circ}$ auf $39,2^{\circ}$. Nach einer bestimmten Zeit wurde mit Aether extrahiert, Aether und unverändertes Amylen auf dem Wasserbade abgetrieben und der Rest rektifiziert. Bereits nach zweistündigem Stehen des Amylens nit Floridin haben sich zirka 15 Proz. über $100^{\circ}$ siedender Polymerisationsprodukte gebildet, nach zwei Wochen betrug die Ausbeute zirka 85 Proz. Das Reaktionsprodukt wurde mit einem kleinen Dephlegmatór von Glins ky rektifizjert, wobei sich folgendes ergab:

\begin{tabular}{|c|c|c|c|c|c|}
\hline $50-1$ & $\begin{array}{r}2,2 \\
30,6\end{array}$ & , & sp & Gew & 0,7 \\
\hline & 31 & $r$ & " & 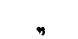 & 0,7 \\
\hline & & $"$ & & & \\
\hline & 26 & & & & 0,8078 \\
\hline
\end{tabular}

5) E. Graefe, Chem. Revue 1910, 3. 
Die Fraktion $150-170^{\circ}$ enfärbt Bromwasser sehr energisch, gibt ein schweres Nitroprodukt mit rauchender Salpetersäure und zeigt auch sonstige Reaktionen des Diamylens. - Aehnliche Polymerisation bewirkt das Floridin auch am Hexylen. Polymerisationsversuche mit Azeton und dergleichen sind im Gange.

Laboratorium

der Naphthaproduktionsgesellschaft Gebr. Nobel, St. Petersburg.

\title{
Zur Kenntnis der Adsorption, III. Kinetik der Entwässerung und Wässerung der Kolloide.
}

\author{
Von Adam Rakowski (Moskau).
}

(Eingegangen am 22. April 1912)

I.

Theoretischer Teil. Die Geschwindigkeit der Adsorption nicht nur des Wassers, sondern auch der im Wasser gelösten Stoffe ist äuberst eigentümlich. Zuerst verlauft die Adsorption sehr schnell, dann fält sie rasch und währt zuweilen längere Zeit. So verdampfen im Falle der Stärke zw'ei Drittel des gesamten Wassers (oder werden adsorbiert) im Laufe der ersten 24 Stunden, das letzte Drittel verliert sich (oder wird adsorbiert) im Laufe von zwei Monaten, wobei auf den zweiten Monat $1 / 50$ des gesamten Wassers kommen. Es ist offenbar, dab die Adsorption vom kinetischen Standpunkt aus zu den komplizierten Reaktionen gehört, d. h. zu solchen, welche nicht durch einfache Gleichungen niederer Ordnung ausgedrückt werden können. Wenn man die Daten der Geschwindigkeiten der Adsorption des Wassers durch ein Kolloid nach den Formeln für die Geschwindigkeiten von monomolekularen Reaktionen berechnet

$$
\begin{aligned}
& K=\frac{1}{t} \ln \frac{a}{x} \text { oder } x=a e^{-K t} \\
& K=\frac{1}{t} \ln \frac{a}{a-x} \text { oder } x=a\left(1-e^{-K t}\right)
\end{aligned}
$$

(worii $K$ der Koeffizient der Reaktionsgeschwindigkeit ist, $\mathrm{x}$ der Gehalt des Wassers im Gel im Moment t, a der Gehalt des Wassers im Moment $t=0$ oder $\infty$ ), so erweist es sich, dab $K$ normal die ganze Zeit hindurch abnimmt. In seltenen Fällen kann die Zunahme von $K$ äuberst unbedeutend sein, dann werden die Formeln (1) und (2) die erste Annäherung zu den wirklichen Formeln sein.

Nehmen wir an, dab ein Gel die Summe von zwei Lösungen darstellt. Es ist sehr wahrscheinlich, daB die beiden Lösungen voneinander abhängig sind. In der allgemeinen Formel kann man die beiden Faktoren (die Geschwindigkeit der Entwässerung und die
Abhängigkeit der einen Lösung von der anderen) mathematisch berechnen, die erhaltenen Gleichungen aber nicht experimentell kontrollieren. Deshalb wollen wir die Aufgabe vereinfuchen indem wir nämlich annehmen, dath in beiden Lösungen unabhängig voneinander das Wasser verdampft oder adsorbiert wird.

Die Geschwindigkeit der Entwässerung. Der Wassergehalt des Gels ist angenommen im Moment $t=0$ a Gramm oder Prozent, wobei auf die erste Lösung $a_{1}$, auf die $z$ weite $a_{2}$ kommen, d. $h . \quad a=a_{1}+a_{2}$; in einem anderen Moment $t$ wird der Wassergehalt des Gels $x=x_{1}+x_{2}$ sein, worin $x_{1}$ auf die erste, $x_{2}$ auf die zweite Lösung kommen. Stellt $K_{1}$ den Geschwindigkeitskoeffizient der Entwässerung (oder Wässerung) der ersten, $K_{2}$ den der zweiten Lösung dar, so können wir schreiben:

$$
\begin{array}{rlrl}
-\frac{d x_{1}}{d t} & =K_{1} x_{1} & -\frac{d x_{2}}{d t} & =K_{2} x_{2} \\
x_{1} & =a_{1} e^{-K_{1} t} & x_{2} & =a_{2} e^{-K_{2} t} \\
& x=a_{1} e^{-K_{1} t}+a_{2} e^{-K_{2} t} &
\end{array}
$$

Die Geschwindigkeit der Wässe. rung. Die Gröben $a_{1}$ und $a_{2}$ haben die früheren Bedeutungen bei $t=\infty$, folglich

$$
\begin{aligned}
& \frac{d x_{1}}{d t}=K_{1}\left(a_{1}-x_{1}\right) \quad \frac{d x_{2}}{d t}=K_{2}\left(a_{2}-x_{2}\right) \\
& x_{1}=a_{1}{ }^{-}\left(1-e^{-K_{7} t_{1}} \quad x_{2}=a_{2}\left(1-e^{-K_{2} t_{1}}\right.\right. \\
& x=a-a_{1} e^{-K_{1} t}-a_{2} e^{-K_{2} t}
\end{aligned}
$$

Die Formeln (3) und (4) erscheinen als die zweite Annäherung $z u$ den wirklichen Formeln, welche die Abhängigkeit der einen Lösung von der anderen ausdrücken mußten. In den Formeln (3) und (4) haben wir drei unbekannte Konstanten $a_{1}, K_{1}$ und $K_{2}\left(a_{2}=a-a_{1}\right)$. Es ist nicht schwer, zu zeigen, daB wir leicht die Ordnung der Bedeutungen $K_{1}$ und $K_{2}$ finden können. Nehmen wir an, daB $K_{\mathrm{J}}$ mehrfach $>K_{\varepsilon}$; bei sehr großen 1-Werten wird das Glied mit 